\title{
A Framework for Efficient Recognition and Classification of Acute Lymphoblastic Leukemia with a Novel Customized-KNN Classifier
}

\author{
Duraiswamy Umamaheswari ${ }^{1}$ and Shanmugam Geetha ${ }^{2}$ \\ ${ }^{1}$ Department of Computer Science, Vidyasagar College of Arts and Science, Udumalpet, Tamil Nadu, India \\ ${ }^{2}$ Department of Computer Science, Government Arts College, Udumalpet, Tamil Nadu, India
}

Even in this modern era today, life's extent is still being challenged by many pathological diseases such as cancer. One such hazard is leukemia. Even a trivial setback in detecting leukemia lead to a severe outcome: the affected cells may eventually prove to be fatal. To combat this, we propose an algorithm to better segment the nucleus region of White Blood Cells (WBC) found in stained blood smear images with the intent of identifying Acute Lymphoblastic Leukemia (ALL). In our proposal, the image is made ready for segmentation in the preprocessing stage by changing its size, brightness, and contrast. In the segmentation stage, the nucleus region is segmented by mathematical operators and Otsu's thresholding. Then mathematical morphological operators are applied in post-processing stage, which makes the nucleus region convenient for feature extraction. Finally, the segmented regions are classified into ALL affected and regular cells by means of the proposed Customized K-Nearest Neighbor classifier algorithm. This work was experimented with over 80 images of the ALL-IDB2 dataset and attained an accuracy rate of $96.25 \%, 95 \%$ of sensitivity and $97 \%$ of specificity.

ACM CCS (2012) Classification: Computing methodologies $\rightarrow$ Machine learning $\rightarrow$ Machine learning approaches $\rightarrow$ Instance-based learning

Computing methodologies $\rightarrow$ Computer graphics $\rightarrow$ Image manipulation $\rightarrow$ Image processing

Applied computing $\rightarrow$ Life and medical sciences $\rightarrow$ Systems biology

Keywords: acute lymphoblastic leukemia, KNN classifier, morphology, segmentation, WBC

\section{Introduction}

The malignancy named leukemia is a type of blood cancer, which spoils the immune system of the human body through the abnormal production of leukocytes. It is categorized into four subtypes: Acute Lymphoblastic Leukemia (ALL), Acute Myelogenic Leukemia (AML), Chronic Lymphoblastic Leukemia (CLL), and Chronic Myelogenic Leukemia (CML). Amongst these, the ALL is a type of childhood cancer [1], but it most frequently affects the elderly population. Timely detection of the disease increases the survival rate of leukemia patients. Although many research proposals have been accepted to accomplish this objective [2] to reduce the death rate, more accurate predictions are needed to early detect the life seizing disease leukemia. This framework has been developed to identify corrupted leukocytes and recognize ALL affected blood cells by segmenting and analyzing the nucleus of leukocytes in microscopic stained blood smear images. In the base work [9], the nucleus region of WBC is segmented using thresholding and morphological operators. Utilization of the threshold value will not accomplish good segmentation with images of varying pixel intensities. In hematology laboratories, the blood smear images are captured in different lightings and atmosphere, so they will be of various levels of brightness. To rectify these issues, we propose segmenting the nucleus region based on enhancing the pixel intensity and contrast, resulting in improved detection accuracy of leukocytes.

This paper is organized as follows: Section 2 shows existing and related research works in the same field, Section 3 produces the methodology of the system proposed, Section 4 discusses the results of experiments made, Section 5 exhibits uniqueness of the system, and Section 6 concludes the paper. 


\section{Literature Review}

Leukemia is a disease of minimal initial symptoms, which makes early detection extremely difficult. But detection at early stage alone saves the life of patients. Many research works in the area of leukemia analysis are done with the major intention of substantiating the burden of hematologists. There are varieties of algorithms for segmenting and classifying blood components such as nucleus, with varying accuracy levels. In 2013, Minal D. Joshi et al. [3] also segmented the nucleus region of WBC by thresholding and morphology, and in their proposal, classification was done by the KNN classifier with $93 \%$ of accuracy.

In 2015, Cecilia Di Ruberto et al. [4] segmented the nucleus of WBCs by means of a machine learning classifier, SVM. In that case, the pixels were segmented through learning by training method, for the sake of ALL identification. Jyoti Rawat et al. [5] reported a classification method using GLCM-texture features and shape features along with the SVM classifier, which shows better classification accuracy rate of $89.8 \%$ than was the one accomplished by applying them separately. Morteza Moradi Amin et al. [6] examined k-means clustering in partitioning the pixels of blood cell images into four distinct clusters, using the $\mathrm{H}$ and $\mathrm{S}$ components of the HSV color space. These clusters represent various constituent parts of blood images. But this does not yield the final segmentation result. In order to obtain the exact region of the nucleus, morphological operators are applied. Subrajeet Mohapatra et al. [7] illustrated that the appropriate combination of image processing and machine learning techniques like c-means clustering algorithm for segmentation, statistical features of nucleus region along with cytoplasm, and ensemble classifiers in classification result in more accuracy in discriminating the types of ALL.

In 2016, C. T. Basima and Janu R. Panicker [8] did the segmentation by k-means clustering and the classification by SVM classifier. They achieved the accuracy rate of $94.56 \%$.

In 2017, Raju Bhukya et al., [9] segmented the nucleus of WBC by thresholding. Then the classification was done with the SVM classifier, which ensured the accuracy rate of $93.33 \%$. This is considered as the base work for this proposal. Jyothi Rawat et al. [10] designed a methodology for segmenting the nucleus of WBC using Otsu's thresholding with morphological operators. In their work, they classified the types of ALL in three phases: classification of normal and cancerous cells, classification of L1 from other ALL types, classification of L2 and L3. Muhammad Sajjad et al. [11] designed a k-means clustering method of segmentation with ensemble multi-class SVM classifier which showed considerable improvement in accuracy rate.

In 2018, Sachin Kumar et al. [12] demonstrated a method of segmentation by k-means clustering with the assistance of color, texture, geometrical and shape features of the nucleus. They used KNN and Naïve Bayes classifiers to achieve effective classification with the accuracy rate of $92.8 \%$. Based on the intensity values, Zeinab Moshavash et al. [13] have separated the leukocytes from erythrocytes and the background of blood smear image. Then ensemble classifiers for classification is done by giving extracted text, shape and LBP features of segmented leukocytes. Here the authors have reported that the LBP features are better for ALL classification than using GLCM. This work has shown the accuracy of $89.91 \%$.

\section{Proposed Framework}

\subsection{Image Acquisition and Preprocessing}

Because of their size, images of the blood cells are seen through microscope only. The input of the proposed system are stained microscopic peripheral blood smear images. There are various freely available datasets of microscopic leukemia images. Amongst them, the standard ALL-IDB benchmark dataset is selected for this investigation.

Processing the obtained raw image is impossible since it can be of any size. Therefore, for the requirement of unity in size, the image is transformed into the conventional size of $256 \times 256$ pixel matrix. In this application, the color information is not needed to extract the edges of WBCs. Thus, to avoid the complexity of visualization and for the amenity of processing, the 3D RGB image is changed into the grayscale image. 
By specifying the smallest and largest stretching limits of intensity values, all the constituent pixels of the image are kept between 0 to 255 . Here the intensity stretching process offers a more prominent show off to the blood cells from their background. Contrast is a vital factor [14] to improve the visual quality of an image. Therefore, the contrast of a blood smear image is improved using the equation (3) mentioned in the algorithm by means of histogram equalization.

\subsection{Image Segmentation}

After the preprocessing phase, the intensity stretched and contrast-enhanced images are joined together. Since the digital images are articulated in terms of matrices of pixels, each element of the first image matrix is added to the matching element in the second image matrix. If the resultant matrix exceeds the range of fractional numbers, they are rounded off. This brightens all the blood components within the image except the nucleus of WBC. Now, each pixel of the brightened image matrix is subtracted by the corresponding pixel in the contrast stretched image to extract the boundaries of blood components. To take out the region of interest (ROI), the pixels of the brightened image are added to the corresponding pixels in the boundary extracted image. As a result, the nucleus region is separated.

Subsequent to partitioning the nucleus region of WBC, the image is filtered using a two-dimensional order statistical minimum filter for removing unwanted noise components that may exist in the image. As a final step of segmentation, the intensity values of the pixels in the filtered gray image is changed to either 0 or 1 valued pixels, based on the Otsu's global thresholding. At this moment, the nucleus region contains 1 valued pixels that are black (in color), the remaining area filled up with 0 valued pixels that are white (in color). Then the image is complemented, to make nucleus and background regions white and appear black in colors correspondingly; this highlights the ROI.

\subsection{Image Post-Processing}

The outcome of segmentation does not give a clear-cut region of the nucleus. Therefore, some post-processing tasks are carried out using mathematical morphological operators. At first, the image is eroded, which discards the boundary pixels for disconnecting the nucleus region from other components. That shrinks the nucleus region a little and unobserved holes inside the region are enlarged. Erosion is done with the help of a structuring element of size 2. To locate the features of the nucleus, it must be a separate and whole component. This is accomplished by the morphological closing operation, which closes the holes at the boundaries of the nucleus region with the same kind of structuring element of size 2 .

Now, the holes inside the region are filled with morphological filling. Filling operation works by changing the connected pixels in the background of ROI to the pixels in the foreground, till the boundaries are reached. A blood smear image may contain more than one WBC-nucleus region. To extract the features of the nucleus for the analysis, the largest of all must be extracted. This is done by labeling and comparing the areas of nucleus regions.

\subsection{Feature Extraction and Classification}

The characteristics of a particular image object are observed by extracting its features. Here, Gray Level Cooccurrence Matrix (GLCM) texture features, statistical and geometrical features of the nucleus are extracted and given as input to Customized K-Nearest Neighbor classifier (C-KNN) [15]. C-KNN classifier measures the distance between the features of ROI, using a customized distance metric, as shown in equation (11). This classifies the given blood cell image into benign or malignant. The process flow of the proposed system is shown in Figure 1.

\subsection{Steps in the Proposed Framework}

\subsubsection{Phase 1}

1. Obtain the microscopic blood smear image to be tested.

2. Resize the obtained true color RGB image into $256 \times 256$ image matrix.

3. Convert the multichannel RGB image into the grayscale image 
Acquire the microscopic blood smear image

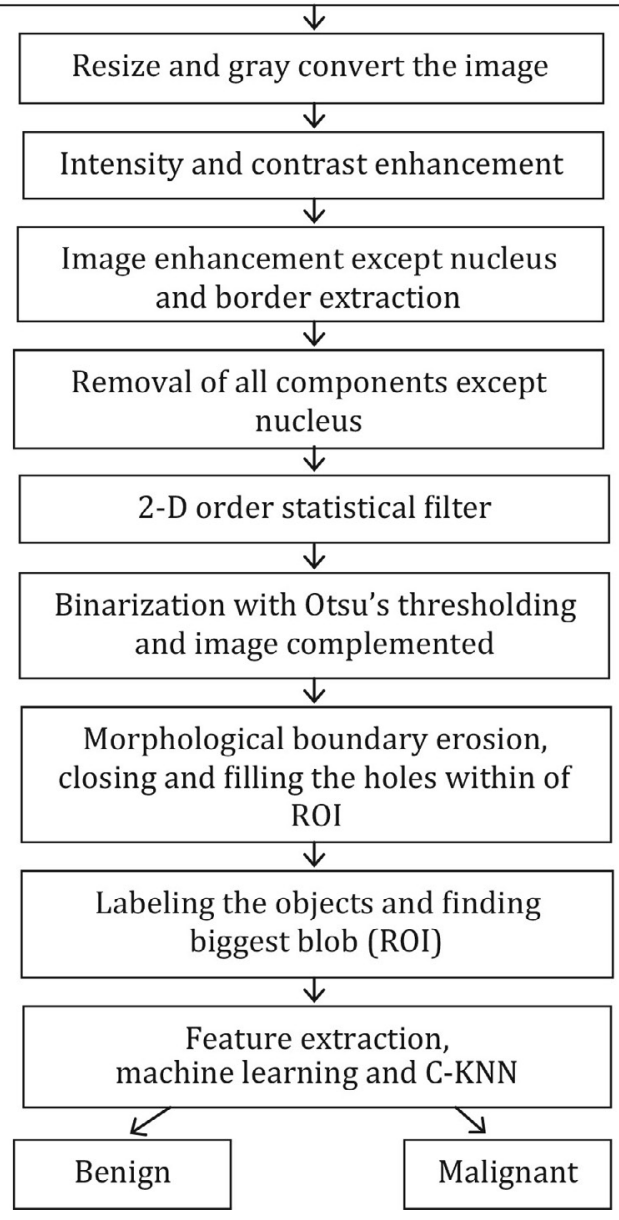

Figure 1. The process flow of the proposed system.

$$
I_{(\text {Gray_Image })}=0.2989 \mathrm{R}+0.5870 \mathrm{G}+0.1140 \mathrm{~B}
$$

4. Amplify the intensity of grayscale image using Linear Contrast Enhancement

$I_{(\text {Intensity_Enhanced })}=$

$=(I-$ Min $) \times \frac{\text { NewMax }- \text { NewMin }}{\text { Max }- \text { Min }}+$ NewMin

5. Adjust the contrast of the image with histogram equalization.

$$
I_{(\text {Contrast_Stretched })}[P(i)]=n_{i} / n, 0 \leq i<L
$$

Here $n_{i}$ is the total number of occurrences of pixel $i$ in the image, $n$ is the total number of pixels in the image, and $L$ is the total number of gray levels in the image.

\subsubsection{Phase 2}

6. Brighten the image, except for the nucleus $I_{1}=I_{(\text {Intensity_Enhanced })}+I_{(\text {Contrast_Stretched })}$

7. Extract the borders of all components.

$$
I_{2}=I_{1}+I_{(\text {Contrast_Stretched })}
$$

8. Separate the nucleus region of WBC

$$
I_{3}=I_{1}+I_{2}
$$

9. Filter the image using 2-D order statistic minimum filtering

$$
\begin{aligned}
& I_{(\text {Filtered_Image })}[P(i)]= \\
& \quad=\operatorname{Min}\left\{I_{X 1}, \ldots, I_{X \mathrm{n}}\right\}, 1 \leq i \leq n ;
\end{aligned}
$$

where $X$ is the window.

10. Binarize the filtered image with the Otsu's global thresholding and complement to highlight ROI.

\subsubsection{Phase 3}

11. Erode boundary of the selected region and expose unseen holes inside ROI

$$
\begin{aligned}
I_{(\text {Eroded_Image })} & =I_{(\text {Filtered_Image })} \theta H \\
& =\left(\bar{I} \oplus H^{*}\right)^{\prime}
\end{aligned}
$$

here, $H$ is the structuring element.

12. Close the selected region by closing

$$
\begin{aligned}
I_{(\text {Closed_Image })} & =I_{(\text {Eroded_Image })} \cdot H \\
& =(I \oplus \mathrm{H}) \theta H
\end{aligned}
$$

where $H$ is the structuring element.

13. Fill the tiny holes within the region

$$
\begin{aligned}
& I_{(\text {Holes_Filled })_{1}}= \\
& \quad=R O I_{K}=\left(R O I_{K-1} \oplus H\right) \cap \mathrm{I}, \text { for } K=1,2, \ldots
\end{aligned}
$$

14. Label the objects in the image to find the biggest blob.

\subsubsection{Phase 4}

15. Extract needed features of the nucleus

16. Classify the images using Customized K-Nearest Neighbor algorithm with the 
distance measure given below:

$$
d=\sqrt{(x+y)^{2}+(x-y)^{2}}
$$

Here, $x, y$ represents the features.

\section{Experimental Results and Discussion}

The proposed method has been experimented with microscopic thin blood smear images acquired from ALL-IDB2 open source benchmark dataset of 260 images of $2592 \times 1944$ pixels resolution [16]. The images have been captured by a Canon PowerShot G5 Camera attached to an optical laboratory microscope. Inside the database, 130 are malignant images of ALL category and 130 are benign images [17]. The dataset is divided into two separate parts, one is a training data set, and the other one is a test data set. The system has learned through 180 images and tested on 80 images. To avoid confusion in the selection, the first 90 images from each malignant and benign category, respectively are chosen for training purpose and the remaining 40 images in each category are used for testing purpose. This gives the results shown in Figure 2, with both malignant and benign image samples.

In leukemia research, mostly texture features of the nucleus region are utilized to define the malignancy. Texture features provide details about the intensity of difference between the pixels. The most important statistical texture extraction method is GLCM. Therefore we analyze the nucleus region based on its visible and invisible characters by means of texture and geometrical features [18]. Table 1 shows the values of 14 GLCM features extracted from the segmented nucleus regions. For illustration, one image is randomly chosen from each, benign and ma-

Table 1. Extracted features of the segmented nucleus region.

\begin{tabular}{|c|c|c|c|}
\hline Feature & Description & Benign & Malignant \\
\hline 1. Contrast & $\begin{array}{l}\text { The measure of intensity between a pixel and its neigh- } \\
\text { bors in the nucleus region. }\end{array}$ & 0.005413 & 0.01647 \\
\hline 2. Homogeneity & Closeness of the distribution of pixels in the region. & 0.9973 & 0.9918 \\
\hline 3. Correlation & The correlation of a pixel to its neighbors in the ROI. & 0.9689 & 0.9529 \\
\hline 4. Energy & Sum of squared elements in GLCM. & 0.8207 & 0.6334 \\
\hline 5. Entropy & $\begin{array}{c}\text { Measure of randomness that characterizes } \\
\text { the texture of ROI }\end{array}$ & 0.4544 & 0.7686 \\
\hline $\begin{array}{l}\text { 6. Root Mean } \\
\text { Square }\end{array}$ & The average value of all pixels in the nucleus region. & 0.0954 & 0.2247 \\
\hline $\begin{array}{l}\text { 7. Standard } \\
\text { Deviation } \\
\end{array}$ & The value of dispersion of a pixel from the mean. & 0.2938 & 0.4174 \\
\hline 8. Smoothness & Smoothness of intensity values in the nucleus region. & 0.9998 & 0.9999 \\
\hline 9. Kurtosis & $\begin{array}{l}\text { Shape of the probability distribution of a real-valued } \\
\text { random pixel. }\end{array}$ & 8.5829 & 2.7405 \\
\hline 10. Skewness & $\begin{array}{l}\text { Asymmetry of probability distribution of real-valued } \\
\text { random pixels. }\end{array}$ & 2.7537 & 1.3193 \\
\hline 11. Centroid & Arithmetic mean position of all pixels inside the region. & 122.13 & 133.37 \\
\hline 12. Area & Total number of pixels inside the region. & 6255 & 14725 \\
\hline 13. Perimeter & Number of nonzero pixels on the boundary of ROI. & 285.92 & 843.97 \\
\hline 14. Roundness & The circularity of ROI. & 0.9665 & 0.2597 \\
\hline
\end{tabular}




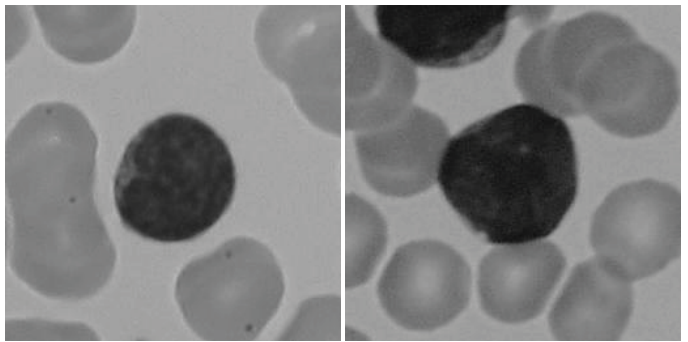

(a) Original images

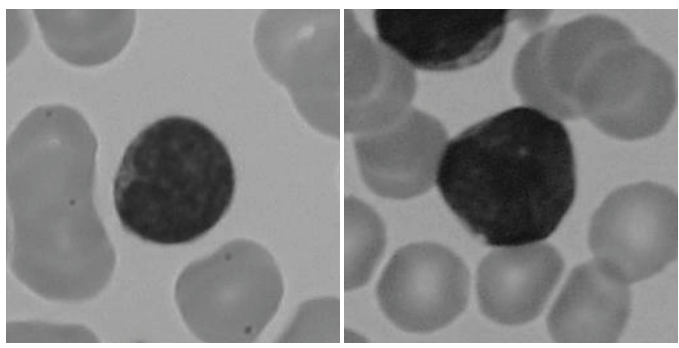

(b) Resized images

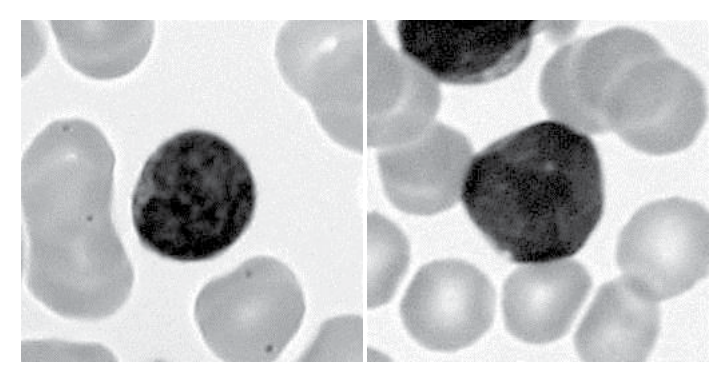

(c) Gray converted and intensity adjusted using LCE

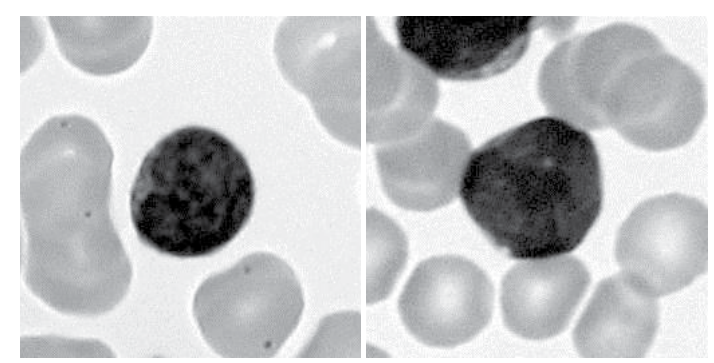

(d) Contrast enhanced

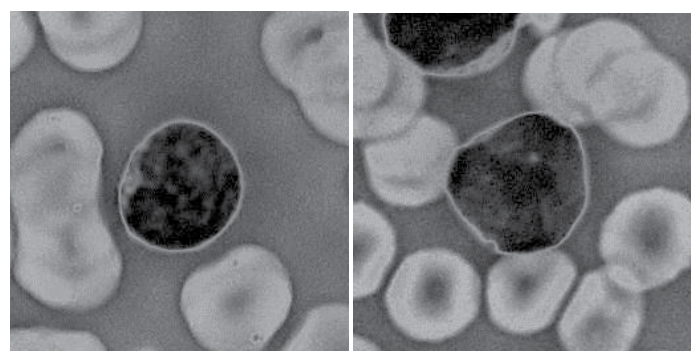

(e). Brightened and boundary extracted.

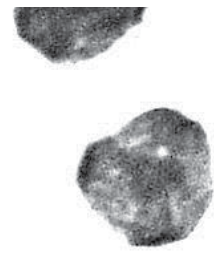

(f) All blood components removed except nucleus
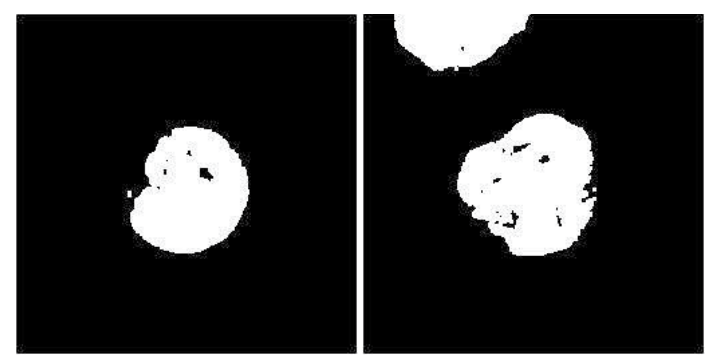

(g) Otsu's thresholded and complemented

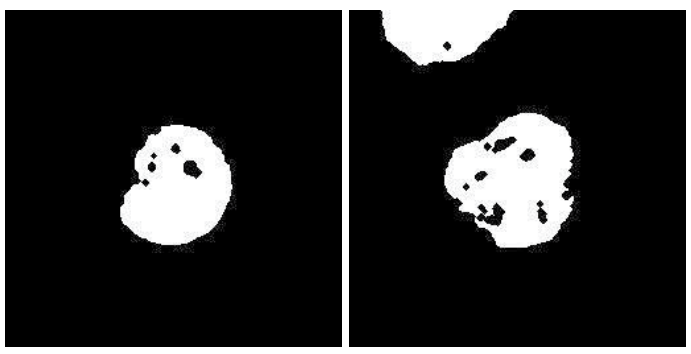

(h) Nucleus eroded images

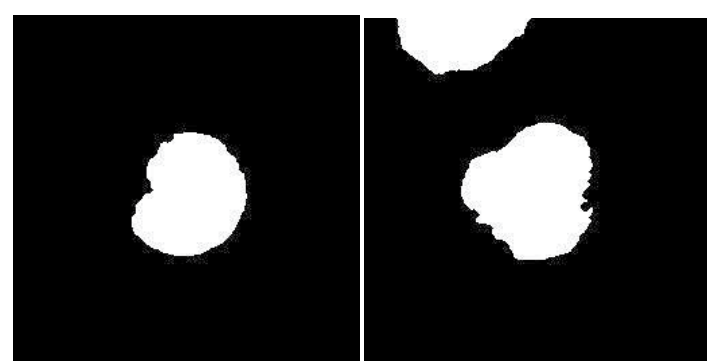

(i) Nucleus regions closed and holes filled-in

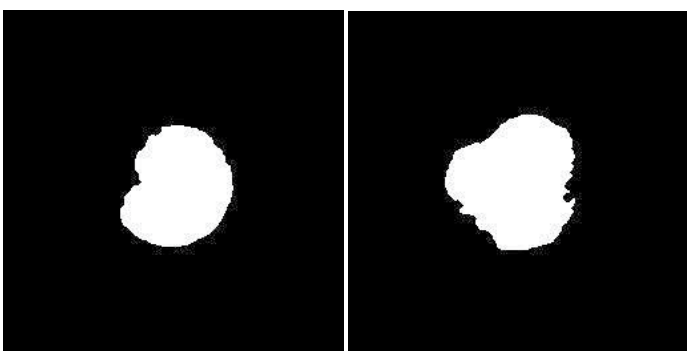

(j). Biggest blob found.

Figure 2. Experimental results of proposed algorithm with benign and malignant images. 
lignant category of the test dataset, and their features are shown. The same set of features are extracted from all the 260 images and then training and testing datasets are selected from them. These features are given as input to the $\mathrm{C}-\mathrm{KNN}$ classifier for classifying cancerous and noncancerous images.

The proposed work is evaluated repetitively on a large number of images. To reduce the computational time of GLCM, the number of gray levels of the image is reduced from 256 to 32 levels by dividing the resized image (12) with an appropriate value [19].

$$
\text { Image }=(256 \times 256 \text { Image }) / 8
$$

By identifying the similarities and variations in the features of 80 test images, the C-KNN classifier has done the classification. Table 2 shows the confusion matrix based on True Positive, False Negative, True Negative, and False Positive values calculated as the results of the classification. Table 3 demonstrates the parameters assessed with the help of the confusion matrix. These parameters facilitate evaluation of the performance of the $\mathrm{C}-\mathrm{KNN}$ classifier.

Table 2. Confusion matrix of the $\mathrm{C}-\mathrm{KNN}$ classifier.

\begin{tabular}{|c|c||c|c|}
\cline { 3 - 4 } \multicolumn{2}{c|}{} & \multicolumn{2}{c|}{ Condition } \\
\cline { 3 - 4 } \multicolumn{2}{c|}{ Positive } & Negative \\
\hline \multirow{2}{*}{ Test } & Negative & True Positive 38 & False Positive 1 \\
\cline { 2 - 4 } & Positive & False Negative 2 & True Negative 39 \\
\hline
\end{tabular}

The receiver operator characteristics curve of the classifier and Area Under the Curve (AUC) are shown in Figure 3 . In the plot, the $X$ coordinate represents the false positive rate and $Y$ coordinate represents the true positive rate. According to the value of AUC, the following judgments can be declared about the classifier performance:

$$
\begin{gathered}
\text { AUC } \geq 0.9 \rightarrow \text { Excellent } \\
\text { AUC }=0.8-0.9 \rightarrow \text { Good } \\
\text { AUC }=0.7-0.8 \rightarrow \text { Fair }, \text { and } \\
\text { AUC }=0.6-0.7 \rightarrow \text { Poor. }
\end{gathered}
$$

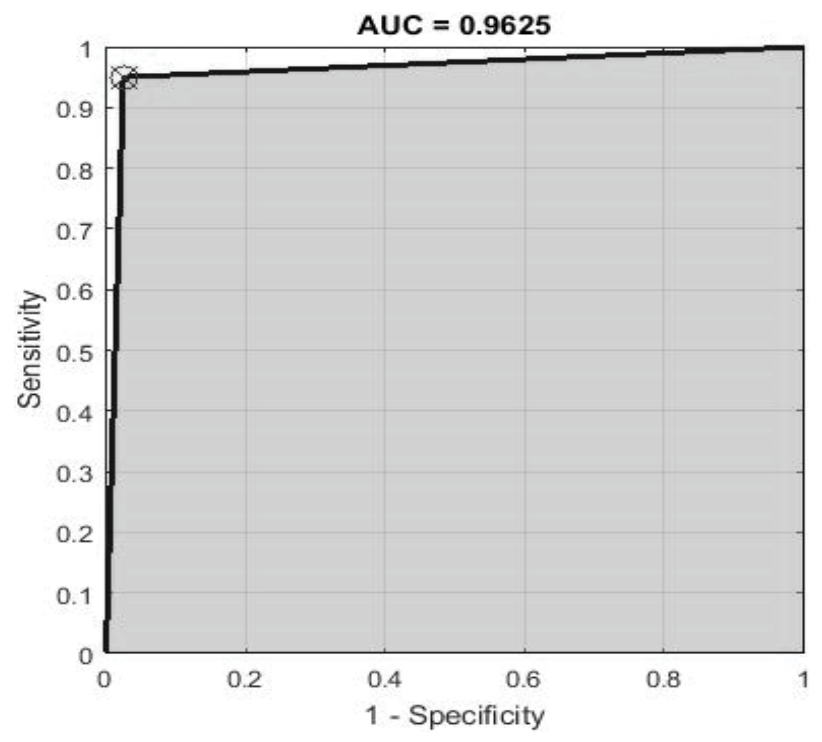

Figure 3. ROC of classification outcome of the binary C-KNN classifier.

Table 3. Performance evaluation from confusion matrix.

\begin{tabular}{|c||c|c|}
\hline Parameters & Formula & Result of Experiments in \% \\
\hline Accuracy & $(\mathrm{TP}+\mathrm{TN}) /((\mathrm{TP}+\mathrm{FN}+\mathrm{TN}+\mathrm{FP}))$ & 96.25 \\
\hline Error Rate & $(\mathrm{FP}+\mathrm{FN}) /((\mathrm{TP}+\mathrm{FN}+\mathrm{TN}+\mathrm{FP}))$ & 3.75 \\
\hline Sensitivity $(\mathrm{TPR})$ & $\mathrm{TP} /(\mathrm{TP}+\mathrm{FN})$ & 95.00 \\
\hline Specificity $(\mathrm{TNR})$ & $\mathrm{TN} /(\mathrm{TN}+\mathrm{FP})$ & 97.50 \\
\hline $\begin{array}{c}\text { Rate of Positive } \\
\text { Predictions }\end{array}$ & $\mathrm{RPP}=(\mathrm{TP}+\mathrm{FP}) /((\mathrm{TP}+\mathrm{FN}+\mathrm{TN}+\mathrm{FP}))$ & 48.75 \\
\hline $\begin{array}{c}\text { Rate of Negative } \\
\text { Predictions }\end{array}$ & $\mathrm{RNP}=(\mathrm{FN}+\mathrm{TN}) /((\mathrm{TP}+\mathrm{FN}+\mathrm{TN}+\mathrm{FP}))$ & 51.25 \\
\hline $\begin{array}{c}\text { False Negative Rate } \\
\text { (FNR) }\end{array}$ & $\mathrm{FNR}=\mathrm{FN} /(\mathrm{TP}+\mathrm{FN})$ & 5.00 \\
\hline $\begin{array}{c}\text { False Positive Rate } \\
\text { (FPR })\end{array}$ & $\mathrm{FPR}=\mathrm{FP} /(\mathrm{TN}+\mathrm{FP})$ & 2.50 \\
\hline
\end{tabular}


The AUC value of the proposed C-KNN classifier is 0.9625 . Hence, its performance can be stated as excellent.

\section{The Significance of the Proposed Framework}

The KNN classifier is a supervised classification method. It determines the class by measuring the distance between the features of training samples and test samples. If the measured distance for a given test image is more similar to a malignant training sample, then the image is classified as malignant, otherwise it is classified as benign. Numerous research works have been implemented with KNN classifier by applying typical distance measures.

Application of different distance metrics creates a greater impact on the classification accuracy [20]. The most commonly used distance measure is Euclidean, since the KNN classifier with Euclidean distance measure (13) performs better than all other standard metrics like Cityblock, Minkowsky, and Cosine.

$$
d=\sqrt{\frac{\sum_{i=1}^{m}\left(x_{i}-y_{i}\right)^{2}}{m}}
$$

As an exploratory approach, the KNN classifier with a new customized distance measure shown in the equation (11) has been experimented in this proposal. It achieves upgraded performance by finding the distance between a test data point and sample data points more accurately [21].

The proposed approach is compared with the performance of a number of other (existing) systems in which standard machine learning classifiers have been used for classification. Amongst these, the proposed C-KNN classifier provides the highest accuracy rate of $96.25 \%$ and also outperforms the base approach. Improved classification accuracy proves that the distance measure we proposed finds more accurate distance with ALL-IDB2 leukemia dataset than the typical distance measures used in existing systems using the same dataset. Hence, in our work, the customization of $\mathrm{KNN}$ is done by applying a novel distance measure. The proposed segmentation algorithm has also been experimented with the KNN classifier with Euclidean measure achieving an accuracy rate of $95.96 \%$.

\section{Conclusion}

Our approach detects the ALL with $96.25 \%$ accuracy rate which is better than in other existing approaches. Hence it will show more accurate predictions with peripheral blood smear images. For doing this, the system segments the nucleus region of WBCs, which are classified based on GLCM texture, statistical and geometrical features. As an entirely novel approach, the Customized K-Nearest Neighbor classifier classifies with $96.25 \%$ accuracy, $95 \%$ sensitivity and $97.50 \%$ specificity. This is $0.29 \%$ more

Table 4. The accuracy of the proposed framework compared to existing approaches.

\begin{tabular}{|c||c|c|c|c|}
\hline Author & Proposed year & $\begin{array}{c}\text { Number of Test } \\
\text { images used }\end{array}$ & Classifier & Accuracy \\
\hline \hline Minal D. Joshi et al., [3] & 2013 & 108 & KNN & $93 \%$ \\
\hline Jyoti Rawat et al.[5] & 2015 & 260 & SVM & $89.8 \%$ \\
\hline C. T. Basima, Janu R. Panicker [8] & 2016 & 94 & SVM & $94.56 \%$ \\
\hline Raju Bhukya et al., [9] & 2017 & 80 & SVM & $93.33 \%$ \\
\hline Sachin Kumar et al. [12] & 2018 & 60 & KNN & $92.8 \%$ \\
\hline Zeinab Moshavash et al. [13] & 2018 & 108 & KNN & $94.76 \%$ \\
\hline $\begin{array}{c}\text { Proposed approach with KNN } \\
\text { (applying Euclidean) }\end{array}$ & - & 80 & KNN-Euclidean & $95.96 \%$ \\
\hline Proposed work & - & 80 & C-KNN & $96.25 \%$ \\
\hline
\end{tabular}


accurate than KNN with Euclidian measure and $2.92 \%$ more accurate than the base work. The AUC value achieved by this approach is 0.9625 . To save people's lives, the proposed approach can be used in hemato-pathology laboratories, in order to assist hematologists in detecting leukemia more accurately. Since this research proposal is producing commendable results, it will act as an initiative for making modifications in the KNN algorithm with some other distance measures. In our future work, we are planning to achieve improvements using the ensemble of different classifiers combined with the proposed system to produce even more accurate results.

\section{References}

[1] C.-H. Pui et al., "Mechanisms of Disease Acute Lymphoblastic Leukemia", The New England Journal of Medicine, vol. 350, pp. 1535-48, 2004.

[2] S. Shafique and S. Tehsin, "Computer-Aided Diagnosis of Acute Lymphoblastic Leukaemia", Computational and Mathematical Methods in Medicine, vol. 2018, 2018.

https://doi.org/10.1155/2018/6125289

[3] M. D. Joshi et al., "White Blood Cells Segmentation and Classification to Detect Acute Leukemia", International Journal of Emerging Trends \& Technology in Computer Science, vol. 2, no. 3, 2013.

[4] C. Di Ruberto et al., "A Multiple Classifier Learning by Sampling System from White Blood Cells segmentation", ResearchGate, Conference Paper, August 2015.

https://doi.org/10.1007/978-3-319-23117-4_36

[5] J. Rawat et al., "Computer Aided Diagnostic System for Detection of Leukemia Using Microscopic Images", 4th International Conference on Eco-Friendly Computing and Communication Systems, Procedia Computer Science, vol. 70, pp. 748-756, 2015.

https://doi.org/10.1016/j.procs.2015.10.113

[6] M. M. Amin et al., "Recognition of Acute Lymphoblastic Leukemia Cells in Microscopic Images Using K-Means Clustering and Support Vector Machine Classifier", Journal of Medical Signals and Sensors, vol. 5, no. 1, pp. 49-58, 2015.

[7] S. Mohapatra et al., "Automated Morphometric Classification of Acute Lymphoblastic Leukaemia in Blood Microscopic Images Using an Ensemble of classifiers", Computer Methods in Biomechanics and Biomedical Engineering: Imaging and Visualization, vol. 4, no. 1, 2016. https://doi.org/10.1080/21681163.2014.897650
[8] C. T. Basima et al., "Enhance Leukocyte Classification for Leukemia Detection", International Conference on Information Science (ICIS), IEEE, 2016, pp. 65-71.

[9] R. Bhukya et al., "Detection of Acute Lymphoblastic Leukemia using Microscopic Images of Blood", International Journal of Advanced and Applied Sciences, vol. 4, no. 8, pp. 74-78, 2017. https://doi.org/10.21833/ijaas.2017.08.011

[10] J. Rawat et al., "Classification of Acute Lymphoblastic Leukaemia Using Hybrid Hierarchical Classifiers", Multimedia Tools and Applications, Springer 2017, 76: 19057 https://doi.org/10.1007/s11042-017-4478-3

[11] M. Sajjad et al., "Leukocytes Classification and Segmentation in Microscopic Blood Smear: A Resource-Aware Healthcare Service in Smart Cities", IEEE ACCESS, 2017. https://doi/10.1109/ACCESS.2016.2636218

[12] S. Kumar et al., "Automatic Detection of Acute Leukemia using K-Mean Clustering Algorithm", Advances in Intelligence Systems and Computing, vol. 554, Springer, 2018. https://doi.org/10.1007/978-981-10-3773-3_64

[13] Z. Moshavash et al., "An Automatic and Robust Decision Support System for Accurate Acute Leukemia Diagnosis from Blood Microscopic Images", Journal of Digital Imaging, Springer, 2018.

https://doi.org/10.1007/s10278-018-0074-y

[14] C. Y. Wong et al., "Image Contrast Enhancement using Histogram Equalization with Maximum Intensity Coverage", Journal of Modern Optics, vol. 63, no. 16, 2016. https://doi.org/10.1080/09500340.2016.1163428

[15] F. Albregtsen, "Statistical Texture Measures Computed from Gray Level Cooccurrence Matrices", CiteSeerX, 2008.

www.uio.no https://homes.di.unimi.it/scotti/all/

[16] R. Donida Labati et al., "ALL-IDB: The Acute Lymphoblastic Leukemia Image Database for Image Processing", 18th IEEE International Conference on Image Processing, 2011, pp. 2045-2048. http://doi.org/10.1109/ICIP.2011.6115881

[17] M. M. Trivedi, "Object Detection Based on Gray Level Cooccurrence", Computer Vision, Graphics, and Image Processing, vol. 28, pp. 199-219, 1984.

http://dx.doi.org/10.1016/S0734-189X(84)80022-5

[18] D.-C. He et al., "Texture Feature Extraction", Pattern Recognition Letters, vol. 6, no. 4, pp. 269-273, 1987. https://doi.org/10.1016/0167-8655(87)90087-0

[19] L.-Y. Hu, "The Distance Function Effect on K-nearest Neighbor Classification for Medical 
Datasets", Springerplus, vol. 5, no. 1, pp. 1304, 2016.

https://doi.org/10.1186/s40064-016-2941-7

[20] S. Arumugam and A. Thangapandi Isaac, "Modern Analysis", New Gamma Publishing House, Palayamkottai, India.
Contact addresses:

Duraiswamy Umamaheswari Department of Computer Science Vidyasagar College of Arts and Science Udumalpet, Tamil Nadu

India

e-mail: ums1082@gmail.com

Shanmugam Geetha

Department of Computer Science

Government Arts College

Udumalpet, Tamil Nadu

India

e-mail: geet_shan@yahoo.com
Received: April 2018

Revised: July 2018

Accepted: July 2018
DuRAISWAMY UMAMAHESWARI has completed her MSc, and MPhil, degrees in Computer Science. She presented three papers in international conferences and published four papers in international journals. She has qualified in State Level Eligibility Test (SLET). At present she is working as Assistant Professor in Vidyasagar College of Arts and Science, Udumalepet, Tamil Nadu. She has 12 years of teaching experience at various colleges. Currently she is pursuing the $\mathrm{PhD}$ (Part-Time) degree in Computer Science, at the Government Arts College, Udumalpet, Tamil Nadu, India. Her area of interest is image processing.

Shanmugam Geetha has completed her MSc, MPhil and PhD degrees in Computer Science. She has published more than ten papers in international journals of repute. Her area of specialization is soft computing. She has 20 years of experience in PSG College of Technology, Coimbatore, Tamil Nadu. Presently she is working as Assistant Professor at Government Arts College, Udumalpet, Tamil Nadu, India. 